\title{
Calibration Techniques for Studying Venus and Mars Atmospheres
}

\author{
Edoardo Gramigna' ${ }^{1}$
}

Received: 16 September 2020 / Revised: 20 October 2020 / Accepted: 26 October 2020 / Published online: 9 November 2020 (c) The Author(s) 2020

\begin{abstract}
The European Space Agency Venus Express mission (VEX) was sent to Venus in 2005 to unveil the unsolved mysteries regarding its atmosphere, the plasma environment and its temperatures. Radio occultation experiments performed by VeRa radio science instrument probed the planet's atmosphere by studying the frequency shift on the radio signal sent by the spacecraft to Earth-based ground stations. This study carries out the calibration of the radio frequencies within a radio occultation experiment in order to correct the main sources of error as: thermal noise, spacecraft clock, spacecraft trajectory, and plasma noise. Any uncalibrated effects will bias the retrieval of atmospheric properties. A comparison of the occultation experiments between Venus and Mars is presented, both from the engineering and scientific point of view, through the analysis of Venus Express and Mars Global Surveyor (MGS) occultations data, highlighting stronger calibrations required for VEX, the extreme, hostile, thick Venus' atmosphere, and a friendly, thin Mars' atmosphere. This investigation analyzes Venus Express data recorded by the NASA Deep Space Network in 2014, and the results are compatible to previous studies of Venus atmosphere with VEX between 2006 and 2009.
\end{abstract}

Keywords Calibration · Radio occultation · Radio frequency $\cdot$ Frequency residuals $\cdot$ Atmospheres $\cdot$ Venus $\cdot$ Mars

\section{Introduction}

Venus has been the prime target in the early interplanetary exploration since it is the planet with the closest approach to Earth. The first radio occultation experiment on Venus was performed in 1967 during a flyby of Mariner V [1-3], and a strong contributor to the understanding of this planet was made by Pioneer Venus Orbiter, Venera, Mariner missions and Magellan, which mapped the entire surface of the planet and its gravity up to the degree and order 180 [4]. Venus revealed similarities in size, density, mass, volume, orbital radius and bulk composition to Earth. However, the missions discovered an atmosphere characterized by extremely high temperatures, pressures, and composition which renders it uninhabitable, pointing out two planets that had evolved very differently. In 2005, the European Space Agency sent the Venus Express mission (VEX) to solve the unanswered questions regarding the past, present and future of Venus. One of the main objectives was the study of the atmosphere,

Edoardo Gramigna

edoardo.gramigna@unibo.it

1 Department of Industrial Engineering, University of Bologna, Via Fontanelle 40, 47121 Forlì, FC, Italy ionosphere and gravity of Venus through the VeRa radio science instrument, using S- and X-band radio links (2.3 and $8.4 \mathrm{GHz}$, respectively) between the spacecraft and Earthbased ground stations [5]. The first VEX scientific results have been presented by Pätzold [6], showing vertical profiles of Venus ionosphere between 100 and $500 \mathrm{~km}$ altitude and a neutral atmosphere between 40 and $90 \mathrm{~km}$ altitude. Several studies have been published by Tellmann, Piccialli, Lee, Ando [7-11] regarding also the dynamics of Venus atmosphere as well as its thermal structure. Nowadays Akatsuki spacecraft from the Japanese Space Agency (JAXA) is continuing Venus' exploration and more missions will be sent there in the near future.

The exploration of Mars started in 1965 with the first flyby of Mariner IV [12], followed by MarinerVI and MarinerVII from NASA. The Soviet Union participated in the early exploration of Mars with Mars 2 and Mars 3 orbiters. Then, in the 1970s, NASA launched the Viking missions, followed by Mars Global Surveyor in 1996 [13]. MGS made global observations of Mars through its scientific payload, and it sounded Mars neutral atmosphere and ionosphere too. MGS and previous missions discovered a very thin Mars atmosphere, which starts at only $40 \mathrm{~km}$ altitude, and it is also characterized by temperatures and pressures which are not 
as high as the ones on Venus [14, 15]. Mars Express, Mars Odissey and Mars Reconnaissance Orbiter continued MGS observations to further expand Mars' atmosphere, climate and weather knowledge [16-18]. These missions highlighted a more habitable environment compared to Venus and, as a result, dozens of spacecrafts as orbiters, landers and rovers (which can survive on Mars but not on Venus due to the high surface temperatures and pressures) have been sent to study the Red Planet. In the next years, two new missions will explore Mars, Mars2020 from NASA and Exo Mars 2022 by ESA-Roscosmos.

The goal of this investigation is to show the main differences and similarities between Venus and Mars atmospheres, both from the engineering and scientific point of view, through radio occultation experiments, with a focus on the calibrations performed on the frequency residuals. Section 2 reports the structure of a radio occultation experiment, the adopted method and the developed atmosphere algorithm, the main parameters, as well as the main assumptions and limitations of this investigation. Section 3 introduces how the radio signal sent by the spacecraft is acquired by the Deep Space Network, and how the time-domain signal is converted in the frequency domain, to obtain the frequency residuals. Section 4 presents the frequency residuals calibration process, and the differences between VEX and MGS calibrations. To conclude, Section 5 shows the main scientific results and the comparison between Venus and Mars atmospheres.

\section{Radio Occultation Method}

Atmospheric radio occultation investigations rely on the detection of the frequency change of a radio signal as it travels through the atmosphere of a Solar System object. This is caused by the refraction and bending of the radio signal in the neutral gas and ionospheric plasma around the target object (see Fig. 1), which modify the frequency of the radio signal, making it different with respect to what expected by the receiver if no occultation would have occurred. In this way the frequency residuals can be evaluated, which are defined as the difference between the modified frequency during occultation and the expected direct frequency without refraction and occultation.

However, the measurements do not contain only the atmosphere information. The propagation of the radio signal is affected by interplanetary plasma along its path (which can not be removed in this investigation, since multiple frequency experiments would be required [19]), as well as Earth's ionosphere and troposphere, and errors related to the spacecraft clock, trajectory, and thermal noise [20]. The calibration process is a crucial step of this work, since it aims to compensate all the noises and errors, in order to obtain

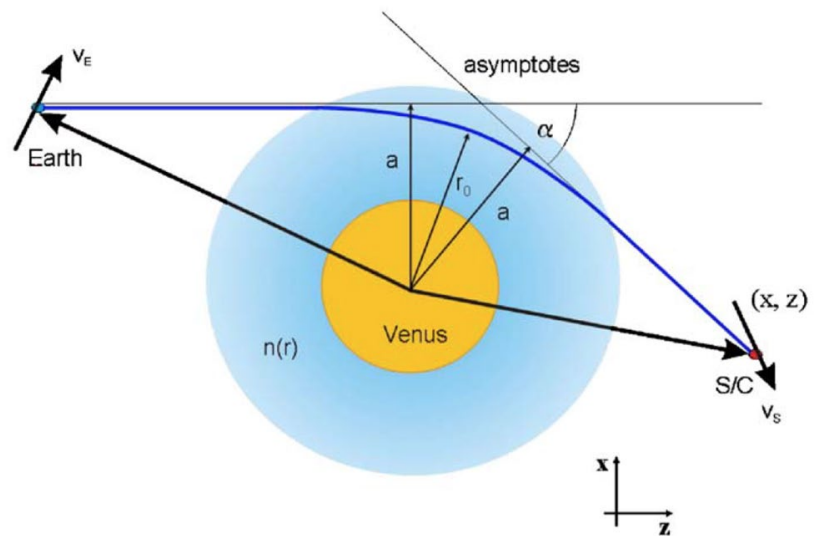

Fig. 1 Ray bending in the atmosphere of Venus. $r_{0}$ is the ray path closest approach distance; $\alpha$ is the bending angle; $a$ is the impact parameter; $n$ is the index of refraction. The radii of the planet and the atmosphere are not to scale. [5]

measurements characterized only by the target's atmosphere information.

Planets' atmospheres are studied through radio occultation data, by implementing the method of Withers [21] within an atmosphere retrieval algorithm, developed in MATLAB environment. In the frame of this investigation, the non-relativistic solution is the one considered, which does not take into account the relativity effects on the radio signal sent by the spacecraft. For both planets, the temperature differences between the relativistic and non-relativistic solution are always lower than $0.5 \mathrm{~K}$, so the relativity effects are negligible. The main assumptions and limitations of this method are:

1. One-way link: the radio signal is transmitted from the spacecraft and received at the ground station;

2. Single frequency: only X-band signals are used in these experiments;

3. Stable frequency source: the source of the transmitted frequency must be stable (use of ultra-stable oscillators by the transmitter is key to the success of one-way link occultations);

4. Spherically symmetric atmosphere and ionosphere at the target: assumption needed to keep the radio signal within the r-z plane defined by Withers (2014) [21], otherwise gradients of refractivity will exists perpendicular to this plane and the radio signal will travel outside the plane;

5. Well-mixed atmosphere: assumption needed to use the hydrostatic equilibrium equation;

6. Ideal gas behavior: the neutral atmosphere is assumed to behave as an ideal gas.

This investigation is based on the atmospheric profiles retrieved from the X-band of both VEX and MGS. The 
VEX data are composed by 25 occultation data (16 egress, 9 ingress) from 2014, recorded by the Deep Space Network (DSN) and managed by the Jet Propulsion Laboratory. On the other hand, a set of 5 MGS occultations (between 1998 and 2006, recorded by the DSN too) are selected for the comparison with Mars' atmosphere. VEX ingress data are mainly concentrated at the north pole, while VEX egress cases, provide a better spatial latitude coverage.

\section{Signal Processing}

Radio occultation experiments rely on their ability to obtain scientific information of a planetary body through the radio frequency of the signal sent by the spacecraft to Earth. Open-loop receivers in the Deep Space Network record the raw antenna voltages of the downconverted signal, relying on frequency predicts to remain tuned to the incoming signal [22]. To get the radio frequencies of the recorded time-domain signal (also called sky frequencies), a spectral fast Fourier transform algorithm (FFT) is adopted, which estimates the main component frequency of the signal for each time step. Then, the sky frequencies are used to evaluate the frequency residuals, which are defined as:

$\Delta f=f_{o}-f_{c}$,

where $f_{o}$ is the sky frequency while $f_{c}$ is the transmitted frequency from the spacecraft corrected for the relative Doppler effects due to the known relative motion of the Earth antenna with respect to the spacecraft, so that only the atmospheric Doppler shifts are measured. These frequency residuals are the input for the atmosphere retrieval algorithm, which converts them in atmospheric parameters of the target body.

The first difference between Venus and Mars, can be seen by looking at the frequency residuals of Figs. 2, 3 . The thick and dense Venus' atmosphere has a stronger effect on the refraction of the radio signal, which result in frequency residuals as high as $5000 \mathrm{~Hz}$, on the other hand the small bending caused by the thin Mars' atmosphere is reflected in residuals of only $1-2 \mathrm{~Hz}$. The frequency residuals are characterized by three main regions: the baseline (between $-2400 \mathrm{~s}$ and $-200 \mathrm{~s}$ of Fig. 2) is the region where the signal is travelling outside the atmosphere of the planet, so the frequency residuals are expected to be flat with a zero mean value; the ionosphere signature (clearly visible at $-100 \mathrm{~s}$ of Fig. 3), whose charged particle advance the Doppler phase of the signal resulting in a positive frequency shift; the atmosphere signature (the slope of the frequency residuals), which represents the signal travelling inside the atmosphere of the planet.

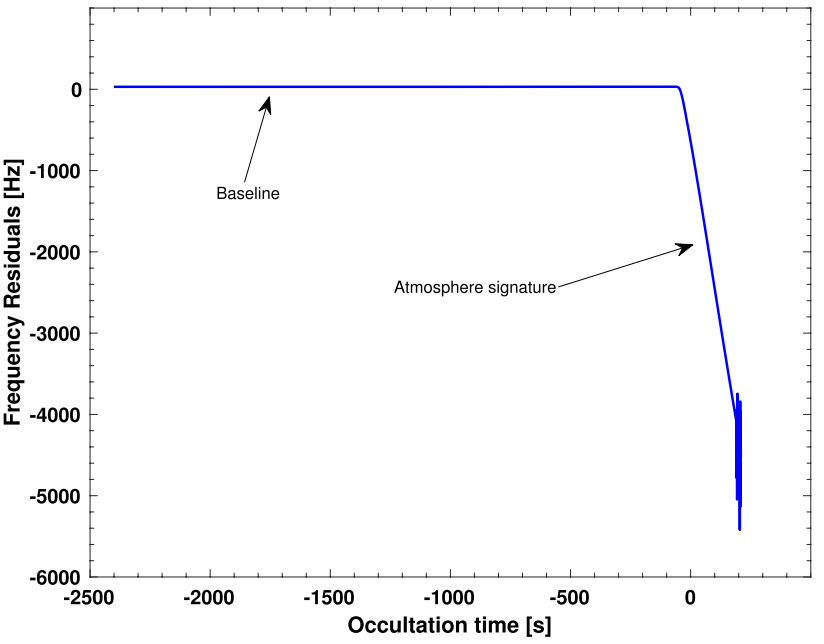

Fig. 2 Frequency residuals from Venus Express, regarding the ingress occultation occurred the 11th March 2014, recorded by the Deep Space Station (DSS)-34. [23]

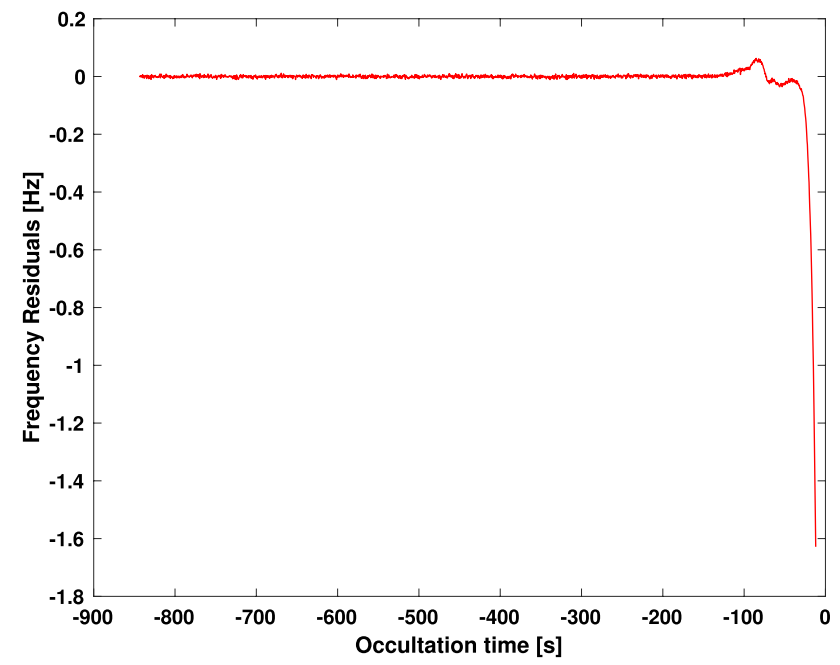

Fig. 3 Frequency residuals from Mars Global Surveyor, regarding the ingress occultation occurred the 27th December 1998, recorded by the DSS-25. [23]

\section{Calibration}

The accuracy of a one-way radio occultation experiment depends on the stability of the transmitted signal frequency. Both VEX and MGS were equipped with an ultra-stable oscillator (USO), an instrument which guarantees a high precise control of the transmitted radio frequency in terms of its Allan Standard Deviation over a wide range of integration times. Both VEX and MGS USO had a frequency stability with an Allan deviation of $10^{-13}$ at integration times 1-100 seconds [5, 14]. However, several noise sources and errors affect the observed frequency 
(i.e. thermal noise, media propagation noise, Earth's ionosphere and troposphere, spacecraft clock and estimated trajectory), which result in fluctuations in the evaluated frequency residuals and, as a consequence, errors in the retrieved atmospheric parameters. The occultation geometry is directly linked to the frequency residuals (see Equation 13 in [21]), which is also analyzed within the Abel transform to compute the refractivity index of the planet's atmosphere, so as a result the retrieved atmospheric parameters will be affected too.

In this work, the order of magnitude of the frequency residual uncertainties is estimated to be: $10^{-3} \mathrm{~Hz}$ for the thermal noise, between $10^{-3}$ and $10^{-4} \mathrm{~Hz}$ for Earth' ionosphere and troposphere (the DSN provides calibrations to remove the delay caused by the Earth's ionosphere to a precision of approximately 2-5 TECU [24]), $10^{-3} \mathrm{~Hz}$ for interplanetary plasma, $10^{-3} \mathrm{~Hz}$ for the spacecraft USO $[25,26]$. The uncertainty due to the estimated spacecraft trajectory, as well as the one of the residual Earth' ionosphere and troposphere particles after calibration are not quantified herein.

For these reasons, the calibration process is crucial to compensate the observed frequency for the noises present in the recorded signal, providing reliable and accurate scientific results. Furthermore, these errors can be easily seen by looking at the baseline of the frequency residuals (see Fig. 4), which in an ideal experiment should be flat with zero-mean value. A zero-mean value baseline indicates that the frequency residuals are fully calibrated for all non-atmospheric sources, so that only the atmospheric refraction signature remains.

The first calibration is performed to correct the frequency residuals for the Earth's ionosphere and troposphere, which cause a frequency delay on the Doppler observables. These corrections are in the form of polynomials whose coefficients

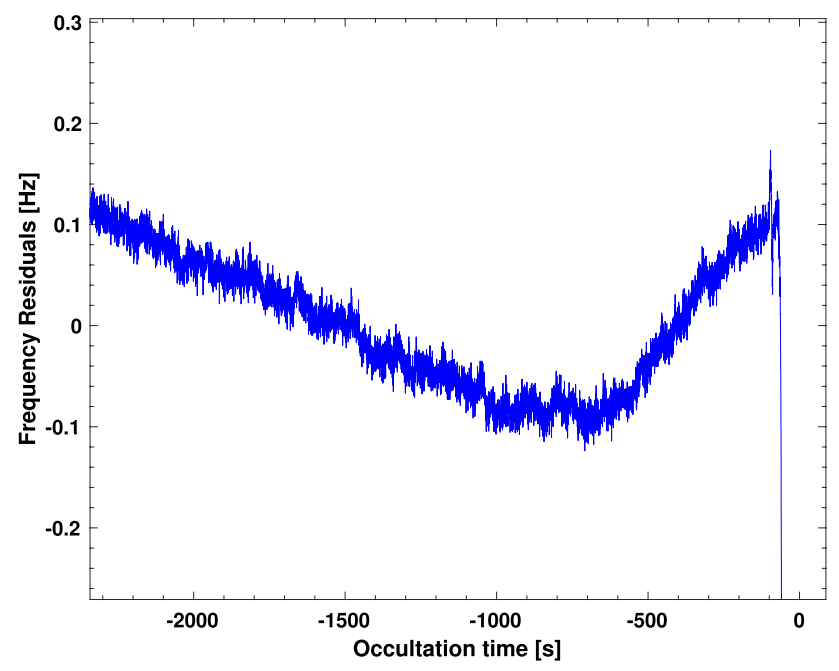

Fig. 4 Baseline of VEX frequency residuals of Fig. 2, not calibrated are provided by the Tracking System Analytic Calibration (TSAC) Group at JPL, based on Global Positioning Systems (GPS) data analysis. In particular, the troposphere polynomial is subtracted from the frequency residuals, while the ionosphere one is added, since the charged particle effect advances the Doppler phase.

Then, a second calibration is required to compensate for the noises errors left as spacecraft clock, estimated trajectory, plasma noise and thermal noise. This is made through a polynomial fitting on the baseline, with the goal to better estimate the transmitted frequency from the unperturbed signal travelling outside the planet's atmosphere. The polynomial coefficients of order $n$ are retrieved from the baseline and they are used to generate a new calibration polynomial for the entire experiment time-span. This polynomial is then subtracted from the frequency residuals to correct the measurements, so that the final calibrated frequency residuals are obtained.

Equation 2 summarizes the entire calibration process:

$\Delta f_{\text {calibrated }}=\Delta f-T+I-\left[p_{1}+p_{2} t+p_{3} t^{2}+\ldots+p_{n} t^{n}\right]$,

where $t$ is the time related to the frequency residuals, $T$ and $I$ are the troposphere and ionosphere calibration polynomials, respectively, and the polynomial of order $n$ corrects for the spacecraft clock, trajectory, thermal noise and plasma noise.

The effect of the calibration on the baseline can be seen by comparing Figs. 4, 5. Even small errors in the knowledge of the trajectory could propagate because of the high dynamics (as gravity) when the spacecraft approaches the planet. Regarding VEX, this is especially pronounced in the ingress region (see Figs. 4, 5, 6) and as a result, higher order polynomials are required for VEX ingress occultations, while VEX egress cases are calibrated with first and second order

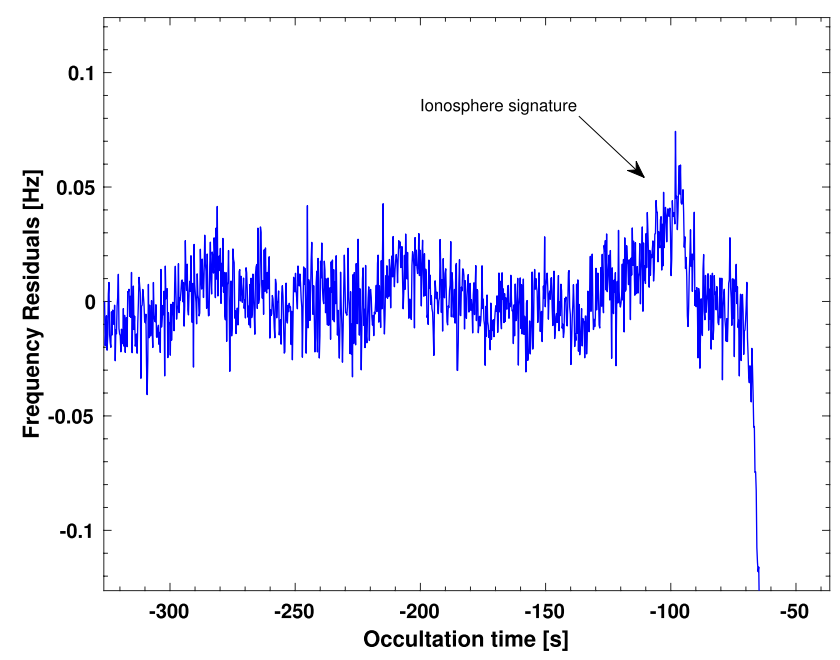

Fig. 5 Calibration on the frequency residuals of Fig. 4 


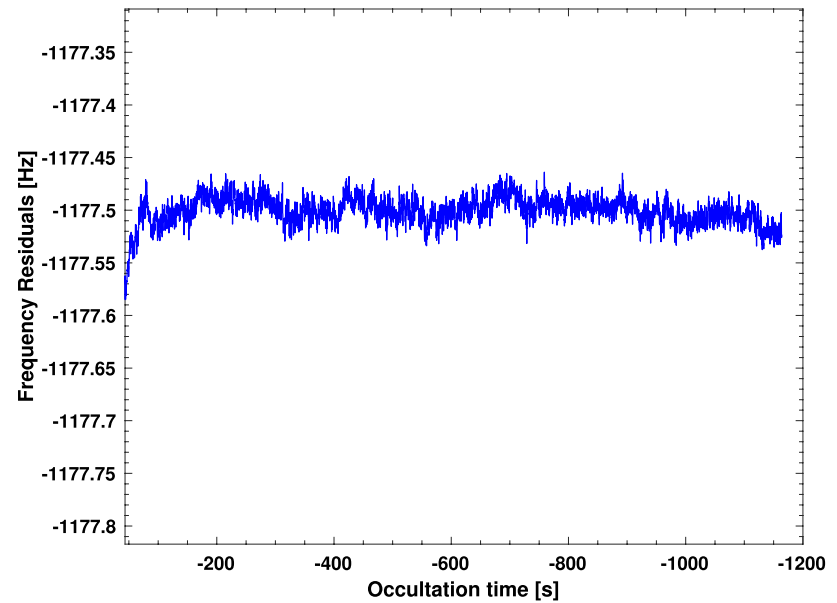

Fig. 6 Baseline of VEX egress occultation on 28th January 2014, not calibrated

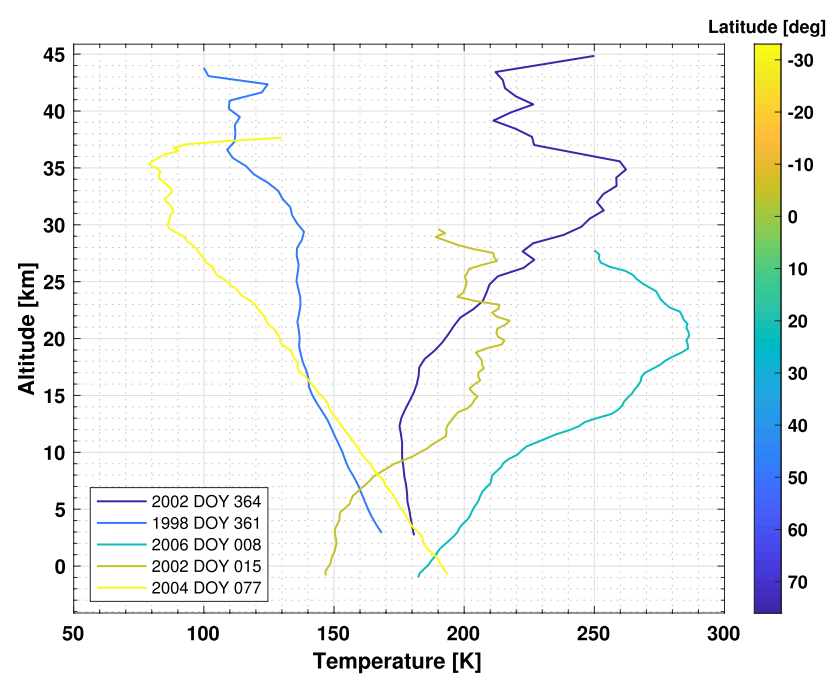

Fig. 7 Temperature profiles for MGS occultations

polynomials. First orders polynomials are applied to MGS occultations, too.

\section{Venus and Mars Atmosphere Comparison}

Venus and Mars atmospheres vertical profiles, retrieved within this work, are presented in Figs. 7, 8, 9. The results clearly show an extreme, hostile environment on Venus, caused by its thick and dense atmosphere, which starts at $100 \mathrm{~km}$ altitude. In addition, the results are limited to $38 \mathrm{~km}$ altitude, due to the critical refraction of Venus atmosphere: the strong bending, refraction, absorption and defocussing of the radio signal at this altitude affects the signal, which becomes so weak that reliable detection is no longer possible

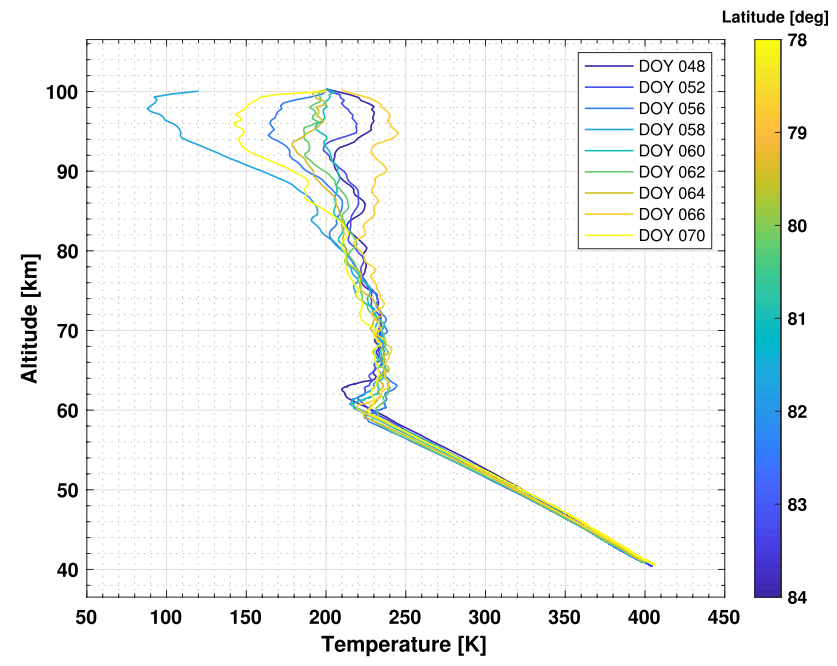

Fig. 8 Temperature profiles for VEX ingress occultations

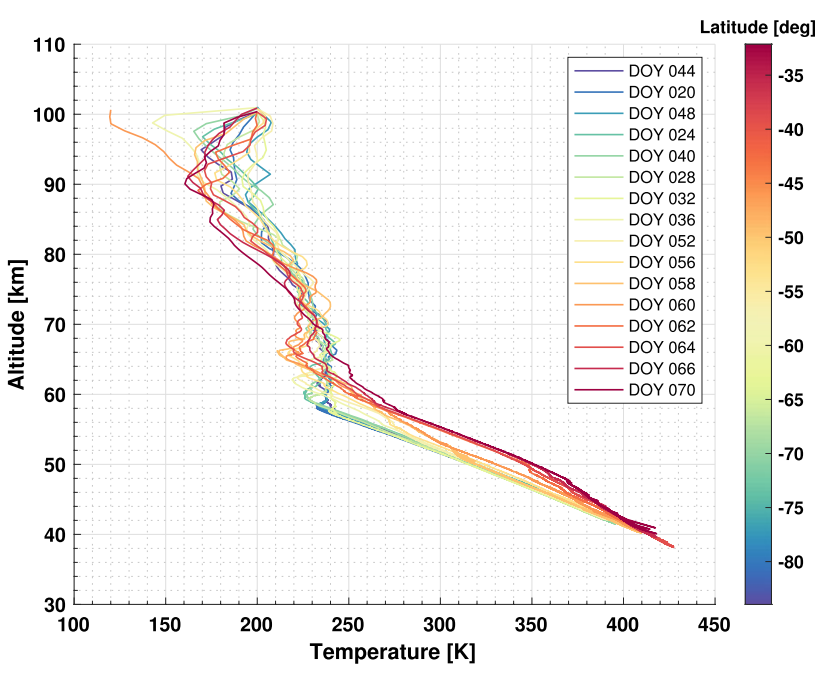

Fig. 9 Temperature profiles for VEX egress occultations

[27]. At this altitude temperatures and pressures are as high as $450 \mathrm{~K}$ and 4 bar (see Figs. 8, 9).

On the other hand, Mars' results present a friendly environment, characterized by a thin atmosphere which starts at $40 \mathrm{~km}$ altitude, temperatures between 150 and $300 \mathrm{~K}$, and extremely low pressure as 0.003 bar at the surface (see Fig. 7). Thanks to its thin and sparse atmosphere, the critical refraction does not occur so that the whole atmosphere, until the Red Planet's surface, can be studied through radio occultation experiments.

Moreover, the results on VEX data are consistent with the ones presented by Tellmann (2009) and Pätzold (2007) $[6,7]$. To conclude, a temperature-latitude dependence is also discovered, resulting in higher temperatures at Venus' equator than at the poles, see Fig. 9. 


\section{Discussion}

This study highlights the calibration of the radio frequencies residuals within radio occultation investigations, a process needed to correct the major sources of noises and errors which affect the frequency residuals measurements. The main process' requirement is related to the polynomial fitting, which must be performed only in the baseline of the frequency residuals, the region where the signal is not perturbed by the target's atmosphere. The calibration process is a crucial part of a radio occultation experiment, and it influences the reliability and the accuracy of the retrieved scientific results. However, to increase the accuracy of this work, the VEX estimated trajectory should be corrected, since the major errors found are attributable to this effect, especially for the VEX ingress cases.

Furthermore, the major differences and similarities between Venus and Mars are presented, both from the engineering and scientific point of view. The similarities are related to the relativity effects, which are negligible for both planets, and the atmosphere retrieval algorithm which behaves in the same way. However, major differences than similarities are discovered. First, in the frequencies calibration process, where higher order polynomials for the VEX residuals are adopted, especially for the ingress case due to the errors in the estimated trajectory and spacecraft clock. Second, all the results enlighten two completely opposite atmospheres. The Venus one, very high, thick, dense, with high pressures and extremely hot, and the Mars one, smaller in height, characterized by lower pressures and temperatures. As a matter of fact, planning a space exploration on Venus is extremely challenging; therefore, this is one of the reasons which explains why in the last decades Mars has been explored more than Venus.

Moreover, Venus throughout the 2014 always shows the same general trend on the retrieved temperature profiles and, in addition, these results are comparable to the ones presented by Pätzold (2007) and Tellmann (2009) [6, 7]. This temperature trend is linked to Venus' high thermal inertia caused by its robust greenhouse effect, which traps the heat inside the atmosphere, but also to its zonal winds which transfer the heat in the mid-low altitudes in an efficient way. Together, these effects, tend to keep Venus' temperature trend unchanged throughout the years. On the other hand, Mars does not experience these phenomena, and the temperature trend undergoes more variations depending on several factors, as the relative position between Mars and the Sun and the day/night condition. To conclude, the analysis on VEX data highlight a temperature-latitude dependence, so that Venus' temperatures at the equator are higher than at the poles.
Acknowledgements This work was partially carried out at the Jet Propulsion Laboratory, California Institute of Technology, under a contract with the National Aeronautics and Space Administration. The author acknowledges the sponsorship of the University of Bologna and the National Aeronautics and Space Administration. Dr. Kamal Oudrhiri, Dr. Marzia Parisi, Dr. Dustin Buccino, Prof. Marco Zannoni, Prof. Paolo Tortora and Dr. Luis Gomez Casajus for their support and their inspiration.

Conflict of interest The author declares that he has no conflict of interest.

Funding Open access funding provided by Alma Mater Studiorum Università di Bologna within the CRUI-CARE Agreement.

Open Access This article is licensed under a Creative Commons Attribution 4.0 International License, which permits use, sharing, adaptation, distribution and reproduction in any medium or format, as long as you give appropriate credit to the original author(s) and the source, provide a link to the Creative Commons licence, and indicate if changes were made. The images or other third party material in this article are included in the article's Creative Commons licence, unless indicated otherwise in a credit line to the material. If material is not included in the article's Creative Commons licence and your intended use is not permitted by statutory regulation or exceeds the permitted use, you will need to obtain permission directly from the copyright holder. To view a copy of this licence, visit http://creativecommons.org/licenses/by/4.0/.

\section{References}

1. Fjeldbo, G., Eshleman, V.R.: Atmosphere of venus as studied with the mariner 5 dual radio-frequency occultation experiment. Radio Sci. 4(10), 879-897 (1969)

2. Fjeldbo, G., Kliore, A.J., Eshleman, V.R.: The neutral atmosphere of venus as studied with the mariner $\mathrm{V}$ radio occultation experiments. Astron. J. 225(2), 1-21 (1971)

3. Barth, C.A.: Venus: Ionosphere and atmosphere as measured by dual-frequency radio occultation of Mariner V. Science 158(3809), 1678-1683 (1967)

4. Konopliv, A.S., Banerdt, W.B., Sjogren, W.L.: Venus Gravity: 180th Degree and Order Model. Icarus 139(1), 3-18 (1999)

5. Häusler, B., Pätzold, M., Tyler, G.L., Simpson, R.A., Bird, M.K., Dehant, V., Barriot, J.P., Eidel, W., Mattei, R., Remus, S., Selle, J., Tellmann, S., Imamura, T.: Radio science investigations by VeRa onboard the Venus Express spacecraft. Planet. Space Sci. 54(13-14), 1315-1335 (2006)

6. Pätzold, M., Häusler, B., Bird, M.K., Tellmann, S., Mattei, R., Asmar, S.W., Dehant, V., Eidel, W., Imamura, T., Simpson, R.A., Tyler, G.L.: The structure of Venus' middle atmosphere and ionosphere. Nature 450(7170), 657-660 (2007)

7. Tellmann, S., Pätzold, M., Häusler, B., Bird, M.K., Leonard Tyler, G.: Structure of the Venus neutral atmosphere as observed by the Radio Science experiment VeRa on Venus Express. J. Geophys. Res. E 114(4), 1-19 (2009)

8. Tellmann, S., Häusler, B., Hinson, D.P., Tyler, G.L., Andert, T.P., Bird, M.K., Imamura, T., Pätzold, M., Remus, S.: Small-scale temperature fluctuations seen by the VeRa Radio Science Experiment on Venus Express. Icarus 221(2), 471-480 (2012)

9. Piccialli, A., Tellmann, S., Titov, D.V., Limaye, S.S., Khatuntsev, I.V., Pätzold, M., Häusler, B.: Dynamical properties of the Venus mesosphere from the radio-occultation experiment VeRa onboard Venus Express. Icarus 217(2), 669-681 (2012) 
10. Lee, Y.J., Titov, D.V., Tellmann, S., Piccialli, A., Ignatiev, N., Pätzold, M., Häusler, B., Piccioni, G., Drossart, P.: Vertical structure of the Venus cloud top from the VeRa and VIRTIS observations onboard Venus Express. Icarus 217(2), 599-609 (2012)

11. Ando, H., Imamura, T., Tellmann, S., Pätzold, M., Häusler, B., Sugimoto, N., Takagi, M., Sagawa, H., Limaye, S., Matsuda, Y., Choudhary, R.K., Antonita, M.: Thermal structure of the Venusian atmosphere from the sub-cloud region to the mesosphere as observed by radio occultation. Sci. Rep. 10(1), 1-7 (2020)

12. Kliore, A., Cain, D.L., Levy, G.S., Eshleman, V.R., Fjeldbo, G., Drake, F.D.: Occultation experiment: Results of the first direct measurement of Mars's atmosphere and ionosphere. Science 149(3689), 1243-1248 (1965)

13. Albee, A.L., Arvidson, R.E., Palluconi, F., Thorpe, T.: Overview of the Mars Global Surveyor mission. J. Geophys. Res. E 106(E10), 23291-23316 (2001)

14. Hinson, D.P., Simpson, R.A., Twicken, J.D., Tyler, G.L., Flasar, F.M.: Initial results from radio occultation measurements with Mars Global Surveyor. J. Geophys. Res. E 104(E11), 2699727012 (1999)

15. Hinson, D.P., Smith, M.D., Conrath, B.J.: Comparison of atmospheric temperatures obtained through infrared sounding and radio occultation by Mars Global Surveyor. J. Geophys. Res. E 109(12), $1-10$ (2004)

16. Fritts, D.C., Wang, L., Tolson, R.H.: Mean and gravity wave structures and variability in the Mars upper atmosphere inferred from Mars Global Surveyor and Mars Odyssey aerobraking densities. J. Geophys. Res. 111(12), 1-14 (2006)

17. Hinson, D.P., Asmar, S.W., Kahan, D.S., Akopian, V., Haberle, R.M., Spiga, A., Schofield, J.T., Kleinböhl, A., Abdou, W.A., Lewis, S.R., Paik, M., Maalouf, S.G.: Initial results from radio occultation measurements with the Mars Reconnaissance Orbiter: A nocturnal mixed layer in the tropics and comparisons with polar profiles from the Mars Climate Sounder. Icarus 243, 91-103 (2014)

18. Tellmann, S., Pätzold, M., Häusler, B., Hinson, D.P., Leonard Tyler, G.: The structure of Mars lower atmosphere from Mars Express Radio Science (MaRS) occultation measurements. J. Geophys. Res. E 118(2), 306-320 (2013)
19. Mariotti, G., Tortora, P.: Experimental validation of a dual uplink multifrequency dispersive noise calibration scheme for Deep Space tracking. Radio Sci. 48(2), 111-117 (2013)

20. Phipps, P.H., Withers, P.: Radio occultations of the Io plasma torus by Juno are feasible. J. Geophys. Res. 122(2), 1731-1750 (2017)

21. Withers, P.L., Moore, K.Cahoy, Beerer, I.: How to process radio occultation data: 1 . From time series of frequency residuals to vertical profiles of atmospheric and ionospheric properties. Planet. Space Sci. 101, 77-88 (2014)

22. Asmar, S.W., Renzetti, N.A.: The Deep Space Network as an Instrument for Radio Science Research. Technical Report 2, NASA-Jet Propulsion Laboratory, Pasadena, CA 91107 (1993)

23. NASA-JPL. Deep Space Network. https://eyes.nasa.gov/dsn/dsn. html. Retrieved 15 Sept 2020

24. Catherine, L.: Thornton and James S. Border. Radiometric Tracking Techniques for Deep Space Navigation, Radiometric Tracking Techniques for Deep Space Navigation (2005)

25. Withers, P.: Prediction of uncertainties in atmospheric properties measured by radio occultation experiments. Adv. Space Res. 46(1), 58-73 (2010)

26. Bocanegra-Bahamón, T.M., Calvés, G.M., Gurvits, L.I., Cimò, G., Dirkx, D., Duev, D.A., Pogrebenko, S.V., Rosenblatt, P., Limaye, S., Cui, L., Li, P., Kondo, T., Sekido, M., Mikhailov, A.G., Kharinov, M.A., Ipatov, A.V., Wang, W., Zheng, W., Ma, M., Lovell, J.E.J., McCallum, J.N.: Venus Express radio occultation observed by PRIDE. Astron. Astrophys. 624, 1-14 (2019)

27. Eshleman, V.R.: The radio occultation method for the study of planetary atmospheres. Planet. Space Sci. 21(9), 1521-1531 (1973)

Publisher's Note Springer Nature remains neutral with regard to jurisdictional claims in published maps and institutional affiliations. 